\title{
A Comparison of Network Simulation and Emulation Virtualization Tools
}

\section{Dr. Te-Shun Chou, East Carolina University}

Dr. Te-Shun Chou is an Associate Professor in the Department of Technology Systems at East Carolina University (ECU). He received his Bachelor degree in Electronics Engineering and both Master's degree and Doctoral degree in Electrical Engineering at Florida International University. His research interests include machine learning, wireless communications, and network security, especially intrusion detection and incident response.

\section{Mr. Steve Keith Baker, East Carolina University}

My teaching interests include networking and security. I also enjoy teaching emerging technology classes and working with the Internet of Things. My background includes twenty years of IT experience with both the private and public sectors. I try to bring real-world examples into my classroom, and provide students with a well-rounded view of expectations in the workforce.

\section{Miguel Vega-Herrera}


A Comparison of Network Simulation and Emulation Virtualization Tools 


\begin{abstract}
Packet Tracer (PT) is an official Cisco software simulator for exercising Cisco network equipment. Graphical Network Simulator (GNS3) is a free emulator software which allows running actual networking software images on a computer. Both provide good Graphic User Interface (GUI) design and have been intensively used for learners to build, configure, and troubleshoot networks in a virtualized network environment. In this paper, PT and GNS3 will be compared from the perspectives of capability and complexity. Strengths and weaknesses of each will be discussed. In addition, networks will be built in order to illustrate the points of our discussion.
\end{abstract}

\title{
1. Introduction
}

As technological advances continue to further integrate different aspects of our lives, it becomes increasingly difficult to not depend on technology on a day to day basis. All of the smart devices, gadgets and gizmos converge at a central point that provides connectivity between them - the network. With the internet boom over the past two decades, network technology has become a crucial discipline that is necessary for technology to truly be useful. Network engineers have to consider a variety of factors, such as bandwidth, traffic type, number of concurrent users, ports needed, cabling, and what hardware to use, when designing a network. Teachers of network concepts need to employ a mixture of devices to create networks to help learners effectively learn network theories and conduct experimental activities, such as setup, configurations, and simulation.

In the past, virtualization technology has been popularly used in designing computer networks. In general, there are two tools that can be used when creating virtualized networks, simulators and emulators. Simulators mimic the basic functions of network devices but do not provide all the properties of the devices, whereas emulators behave exactly like a realistic network. Moreover, in an emulated network, virtual computer system with various operation systems, such as Windows and Linux, could be connected to network devices, routers and switches, in the created virtual network.

There are two well-known network simulation and emulation tools that are widely used in teaching network computer courses. They are PT (Packet Tracer) and GNS3 (Graphical Network Simulator). Each has different features and are Windows and Linux supported. PT is a proprietary network simulator designed by Cisco Networking Academy [1]. It implements only limited Cisco proprietary network devices. The latest version, PT 6.2 is available as a free download to Networking Academy members. GNS3 is an open source network emulator that can be downloaded free [2]. Based on Dynamips, Dynagen, and Qemu, GNS3 is capable of building realistic virtualized networks. Although two tools are similar in design, both programs target different aspects of network design and offer varying levels of functionality. The main goal of this paper is to compare the capabilities of these two popular simulation tools.

This paper is organized as follows. Section 2 discusses the differences between PT and GNS3. Section 3 presents the conclusions. 


\section{Comparison}

In this section, PT will be compared with GNS3 in terms of their functionalities, capabilities, and design.

\subsection{Graphical user interface (GUI) design}

Both PT and GNS3 are interactive tools that provide an intuitive GUI for learners to design and configure virtual network devices. GNS3 has one workplace for placing virtual network devices. PT's interfaces offer two types of Workspace: logical workspace and physical workspace [3]. The logical view allows users to interconnect and interact with network devices, such as routers, switches, and hosts, in order to build a network. The latter provides users the capability to incorporate a design methodology across buildings or geographical boundaries. In addition PT offers two modes of operation, real-time and simulation. In real-time mode, the user experience is close to working with actual equipment in both operation and configuration. Simulation mode enables users to view network traffic in a stepped fashion with control over playback. This mode also provides for inspection of the types of data that would be included within packets and frames traversing the network.

\subsection{Memory requirement}

One of the major benefits of PT is that it's a relatively small program that uses a low amount of resources. For running PT 6.2, the minimum Random-access memory (RAM) requirement is only $512 \mathrm{MB}$, and it is $1 \mathrm{~GB}$ for optimal performance [4]. On the contrary, GNS3 requires a larger memory usage since all network components are being emulated and there must be enough resources to run the program. At least $1 \mathrm{~GB}$ RAM will be required to operate the base program [5]. However, this will vary depending on the amount of virtual machines, routers, switches, etc. are added to a particular emulation scenario. The host machine should have enough storage space and a faster processor to support a medium to large network. For example, running two machines with Windows operation system would require the host machine to have at least 2 GB RAM.

\subsection{Supported network routers and switches}

PT is unique in that, since it is a Cisco product, it natively incorporates much Cisco's technology. It includes many hardware devices such as routers, switches, access points, and as of version 6.2 even cell towers [6]. Since GNS3 is not a native Cisco application, it does not fully integrate with all of Cisco's technology. Currently GNS3 supports only Cisco routing platforms 1700, 2600, 2691, 3600, 3725, 3745, and 7200, Cisco PIX firewalls, Cisco ASA firewalls, and Cisco IDS sensors [5]. Moreover, GNS3 does not support Cisco switch images. The only way to emulate a switch is to use its default generic switch or a router with virtual Cisco EtherSwitch module (e.g. NM-16ESW module). GNS3 is also limited in the Cisco IOS images that are supported. There are numerous images that, they work on real equipment, they are not supported within the same device model within GNS3. This can cause issues with advanced features, such as IPv6, for example. Features and functionality are reduced, and in some cases, desired functionality is non-existent due to the inability of GNS3 to incorporate the latest IOS builds. In addition, to download a Cisco image requires a valid service contract associated to a Cisco 
profile, or it requires paying hundreds of dollars to purchase. Additionally the Cisco Right to Use license is not clear on the virtualization of any Cisco IOS image within the GNS3 environment, regardless of ownership. If neither option applies to a user, then acquiring images becomes very difficult which might prevent GNS3 to be used to its full potential. As for other network operating systems, GNS3 supports the implementation of the Operating System for Juniper routers [7], JunOS, on a VM since it is based on an Unix-like operating system, FreeBSD [8]. At this date, JunOS versions for Juniper M series are known to work in GNS3.

\subsection{Supported protocols}

Both PT and GNS3 are used to help train learners in both fundamental networking principles and complex routing protocols. In design PT and GNS3 are extremely beneficial for network simulation, which enables an engineer to simulate a network environment that closely mimics a production network. Since GNS3 is an emulation software that uses real Cisco IOS Images and can connect the simulated devices to virtual computer systems, depending on the IOS image deployed most protocols in different network layers could be supported. On the other hand, PT only supports a subset of protocols that is shown in Table 1 [9].

Table 1. PT Supported Protocols

\begin{tabular}{|l|l|}
\hline Layer & PT Supported Protocols \\
\hline Application & $\begin{array}{l}\text { FTP , SMTP, POP3, HTTP, TFTP, Telnet, SSH, DNS, DHCP, NTP, } \\
\text { SNMP, AAA, ISR VOIP, SCCP config and calls ISR command support, } \\
\text { Call Manager Express }\end{array}$ \\
\hline Transport & TCP and UDP, TCP Nagle Algorithm \& IP Fragmentation, RTP \\
\hline Network & $\begin{array}{l}\text { BGP, IPv4, ICMP, ARP, IPv6, ICMPv6, IPSec, RIPv1/v2/ng, Multi- } \\
\text { Redistribution, Multilayer Switching, L3 QoS, NAT, CBAL, Zone-based } \\
\text { policy firewall and Intrusion Protection System on the ISR, GRE VPN, } \\
\text { IPSec VPN, HSRP, CEF }\end{array}$ \\
\hline $\begin{array}{l}\text { Network Access / } \\
\text { Interface }\end{array}$ & $\begin{array}{l}\text { Ethernet (802.3), 802.11, HDLC, Frame Relay, PPP, PPPoE, STP, } \\
\text { RSTP, VTP, DTP, CDP, 802.1q, PAgP, L2 QoS, SLARP, Simple WEP, } \\
\text { WPA, EAP, VLANs, CSMA/CD, Etherchannel, DSL, 3/4 G network } \\
\text { support }\end{array}$ \\
\hline
\end{tabular}

\subsection{Supported commands}

Since GNS3 is an emulation software that uses real Cisco IOS Images, it has all the commands available to the specific installed IOS, and behaves as a real router would. On the contrary, PT is a software based simulator that only supports limited routing commands. More advanced optional parameters with commands may not be available. For example, PT has limitation in supporting the full suite of IPv6 commands. In addition, there are limited debugging capabilities, and often the output of extant debugging tools is limited and generic in nature. The environment is often limited to basic implementation of networking features and limited internal dynamic routing protocols, offering little to no support for external protocols such as BGP. Additionally commands that need access to external services may be limited to local testing only, such as the inability to implement AAA security to an external RADIUS or TACACS server. 


\subsection{Supported computer systems}

As a network simulator, PT is very easy to use and does everything it needs to correctly. However, it focuses only on the networking portion of a network, where there still exists a variety of other components, such as PCs, servers, IP phones and smart devices. PT simulates these hardware components but functionality is limited to basic features. It does not include the real software that might be needed in a real-time production environment. Whereas PT emphasizes routing and switching connectivity, GNS3 has the added benefit of testing actual services and applications. With the help of Oracle VirtualBox [10], virtual machines running real OS could be connected to routers and switches created inside of GNS3. In an example highlighting many of its features, a network administrator could have a Redhat Linux Virtual Machine (VM) running an Apache webserver. The administrator could then attach this VM to a Cisco Adaptive Security Appliance's (ASA) Demilitarized Zone (DMZ) and configure it to allow external HTTP connections, then test for this connectivity from a machine outside the network. This particular scenario could be carried out without ever having to actually touch the production network, and allows for testing and troubleshooting before actual implementation.

\subsection{Traffic analysis}

PT is a closed simulation environment used to model and visualize the behavior of a network. In simulation mode, it can capture and display the contents of packets as they travel through the network. This helps learners understand the fundamental concepts of data transfer and propagation across a network. GNS3 itself does not have the facility to analyze data traffic or network behavior in real time, however it can work with network analyzers, such as Wireshark [11], to collect traffic between emulated devices. By using Wireshark on a VM, GNS3 can capture packets on virtual Ethernet or serial interfaces. Then the traffic could be recorded for future analysis.

\subsection{Import network topology between PT and GNS3}

Currently it is not possible to import a PT topology to GNS3 (or vice versa). This is due to the fact that both applications are created by different software vendors and they are not meant to integrate with one another. Another factor is that the devices that are supported differ between both applications. In GNS3 you must specifically add all the IOS images for the devices you plan to use however they are automatically included with the PT base install.

\subsection{Cable connection}

The connection of PT includes crossover cable, straight through cable, fiber cable, phone cable, coaxial cable, serial DTE, serial DCE and console cable [12]. GNS3 also supports numerous connectivity options, such as serial DCE/DTE, however it is not as obvious how to implement such technologies. As well, implementing the physical components in GNS3 requires more effort and is not as intuitive. PT on the other hand provides a click and point environment and provides a more realistic looking Layer 1 experience. The graphical representation of devices is more lifelike, and the user is provided a better experience of working with actual equipment. Devices that are supported are depicted as they would appear in actual hardware. Additionally the method of 
installing module cards to provide enhanced functionality provides the user a much more lifelike experience. GNS3 puts more focus on network components aside from physical representation of actual equipment. GNS3 also offers various Layer 1 connectivity options, but it is not as interactive as the environment provided within PT, nor is it as representative of real equipment.

\subsection{Certification}

Both of the environments are helpful for practicing for industry recognized certifications. PT is more aligned with Cisco certifications, but could be used for other general networking concepts that apply to certs such as CompTia Network+. The simulation mode of operation is a valuable tool to help the learners understand the path of a frame and/or packet. For example, in simulation mode we can inspect the headers of frames, and see how the source and destination MAC addresses are changed, but the IP addresses remain unchanged, as a packet is forwarded across routing domains. We can also see how a PC may formulate a broadcast frame and forward that throughout a broadcast domain when a destination hosts IP address needs to be resolved to a MAC address. These are foundational concepts that are applicable across networking in general, and thus they will assist the learner to understand these concepts. This knowledge can then be transferred to numerous certification paths, aside from Cisco.

GNS3 can also be used to enable aspiring network engineers to practice for certification exams, and do so in a more advanced and feature rich environment. Although it does not offer a simulated packet inspection environment, it does support much more advanced commands and environments. Therefore it is applicable to many other networking certifications beyond Cisco. As well, GNS3 supports virtual machines and connections to the host system's network stack. With these advanced features, numerous other certification tracks could be explored. For example, a user might set up an AAA Radius server on one virtual VM, and a client on another. Being able to network those through a GNS3 simulated network environment offers the learner a more realistic environment. With access to a more feature rich environment, a learner could study for a more advanced certifications, such as the CompTia Advanced Security Practitioner. Another example might include studying for the Redhat RHCSA. With the VM support, a learner could easily set-up multiple Linux VMs and network them through GNS3. This would provide an advanced topology design, and enable a learner to practice networking commands between pairs of the virtual Linux machines. Though GNS3 set-up may be a bit daunting at first, once the topology is in place it does open up a whole new world of possibilities and an endless number of opportunities for advanced study.

PT is an excellent resource for teaching foundational networking topics, and directly supports the necessary commands for preparation in numerous Cisco certification tracks, including CCENT, CCNA, partial CCNP and CCNA Security. GNS3 on the other hand offers additional support for connectivity to other vendors' networking equipment, as well as virtualized hosts and servers of various flavors. GNS3 is a bit more difficult to set up at first, but once this is mastered it opens up opportunities to study for a wealth of certifications. 


\subsection{License}

PT is a stand-alone product, and therefore the only licensing concern is membership in (or alumnus) the Cisco Networking Academy. GNS3 requires access to actual IoS files for the device desired for implementation. This could pose issues with Right to Use, as well as availability and access to the same.

\subsection{Support for the instructor}

PT offers an assessment environment entitled the 'Activity Wizard', which is non-existent in GNS3. With this environment, an instructor is able to 'author customized, guided activities that provide immediate feedback' to the learner. These custom activities can also automate grading routines and facilitates unique testing environments with variable content generation [3]. This is a powerful tool that enables the instructor to create unique assignments, and facilitates for quick and efficient grading of complex configurations.

\section{Conclusions}

Instead of having to choose using either PT or GNS3 for network development, it might be an even better idea to use them both together. As previously discussed, both programs target distinct aspects of an overall project, and can be used to complement each other. PT can be used to create a networking plan that incorporates the internal and external functions of a network. PT could further facilitate ease of assessment and automated grading. GNS3, on the other hand, can focus on the specific hosts that will be running and testing to see if the network logic will suit the intended purpose. PT offers a much faster and easier experience for beginning network technicians. PT offers a much cleaner out-of-the box experience, and could limit the frustration factor for beginning learners. Once learners have mastered the basics, they would be better prepared and more experienced to move into the more complex GNS3 environment. In conclusion, PT and GNS3 should be seen as collaborative tools that can integrate with one another towards a common goal, instead of competing applications. They are both invaluable programs for learners who want to build their computer networking knowledge.

\section{References}

1. Why Learn with Packet Tracer. Retrieved from https://www.netacad.com/about-networkingacademy/packet-tracer/

2. GNS3. Retrieved from http://www.gns3.com/

3. Cisco Packet Tracer. Cisco Networking Academy. Retrieved from http://www.cisco.com/c/dam/en_us/trainingevents/netacad/course catalog/docs/Cisco_PacketTracer_DS.pdf

4. Cisco Packet Tracer 6.0.1 Frequently Asked Questions. Cisco Networking Academy. Retrieved from http://www.slideshare.net/sontrinh1276487/ciscopackettracer601fa-qs

5. GNS3 Community. Retrieved from https://community.gns3.com/community

6. Packet Tracer 6.2 available for download. Retrieved from http://www.packettracernetwork.com/features/packettracer-6-2.html

7. Juniper Routers. Retrieved from http://www.juniper.net/us/en/products-services/routing/ 
8. Freebsd. Retrieved from https://www.freebsd.org/

9. Cisco Packet Tracer. Cisco Networking Academy. Retrieved from https://www.netacad.com/ (requires valid Cisco Netacad login to access)

10. VirtualBox. Retrieved from https://www.virtualbox.org/

11. Wireshark. Retrieved from https://www.wireshark.org/

12. Packet Tracer Wiki. Retrieved from http://packettracer.wikia.com/wiki/Connections 